\title{
Msn5p Is Involved in Formaldehyde Resistance but Not in Oxidative Stress Response in the Methylotrophic Yeast Candida boidinii
}

\author{
Zhenyu Zhai, ${ }^{1}$ Hiroya Yurimoto, ${ }^{1}$ and Yasuyoshi SaKaI ${ }^{1,2,3,}$ \\ ${ }^{1}$ Division of Applied Life Sciences, Graduate School of Agriculture, Kyoto University, \\ Kitashirakawa-Oiwake, Sakyo-ku, Kyoto 606-8502, Japan \\ ${ }^{2}$ Research Unit for Physiological Chemistry, Center for the Promotion of Interdisciplinary Education and Research, \\ Kyoto University, Kitashirakawa-Oiwake, Sakyo-ku, Kyoto 606-8502, Japan \\ ${ }^{3}$ CREST, Japan Science and Technology Agency, 5 Sanban-cho, Chiyoda-ku, Tokyo 102-0075, Japan
}

Received September 8, 2011; Accepted November 13, 2011; Online Publication, February 7, 2012

[doi:10.1271/bbb.110679]

\begin{abstract}
Methylotrophic yeasts, which can utilize methanol as sole carbon and energy source, are exposed to two toxic metabolic intermediates, formaldehyde and hydrogen peroxide, during growth on methanol. Here we report that Msn5p, an importin- $\beta$ family nuclear exporter, participated in the formaldehyde resistance mechanism but not in the hydrogen peroxide resistance mechanism in Candida boidinii. Disruption of the MSN5 gene in this yeast caused retardation of growth on formaldehydegenerating growth substrates such as methanol and methylamine, but the expression levels of the methanolmetabolizing enzymes did not fall. The Msn5p-depleted strain was sensitive to formaldehyde but not to hydrogen peroxide. Furthermore, a yellow fluorescent protein-tagged Msn5p was diffuse in the cytoplasm of C. boidinii when the cells were treated with high concentrations of formaldehyde or ethanol, but was predominantly associated with the nuclei following treatment with hydrogen peroxide.
\end{abstract}

Key words: formaldehyde; hydrogen peroxide; MSN5; Candida boidinii; Pichia pastoris

Formaldehyde (HCHO) and hydrogen peroxide $\left(\mathrm{H}_{2} \mathrm{O}_{2}\right)$ are both highly reactive compounds that have toxic effects on all organisms. Hence all organisms have cellular functions to cope with and detoxify these compounds. In the methanol metabolism of methylotrophic yeasts, which are capable of utilizing methanol as sole carbon and energy source, $\mathrm{HCHO}$ and $\mathrm{H}_{2} \mathrm{O}_{2}$ are initially generated by oxidation of methanol. ${ }^{1-3)}$ Methylotrophic yeasts, such as Pichia pastoris, Hansenula polymorpha, and Candida boidinii, are widely used in the production of heterologous proteins. ${ }^{4-8)}$ Because accumulations of $\mathrm{HCHO}$ and $\mathrm{H}_{2} \mathrm{O}_{2}$ can affect cell growth and protein productivity, it is necessary to elucidate cellular strategies to minimize the toxicity of these compounds.

In methylotrophic yeasts, methanol is first oxidized to $\mathrm{HCHO}$ by alcohol oxidase (AOD) within peroxisomes, resulting in the formation of $\mathrm{H}_{2} \mathrm{O}_{2}$, which is decomposed by peroxisomal catalase. ${ }^{1)}$ A peroxiredoxin, peroxisomal glutathione peroxidase (Pmp20), is responsible for the elimination of alkyl hydroperoxide species derived from $\left.\mathrm{H}_{2} \mathrm{O}_{2}{ }^{9}{ }^{9}\right) \mathrm{HCHO}$ is a central intermediate, located at the branch point between the assimilation and the dissimilation pathway. In the assimilation pathway, fixation of $\mathrm{HCHO}$ with xylulose-5-phosphate into dihydroxyacetone and glyceraldehyde-3-phosphate is catalyzed by dihydroxyacetone synthase within the peroxisomes. On the other hand, $\mathrm{HCHO}$ is oxidized to $\mathrm{CO}_{2}$ by a glutathione (GSH)-dependent $\mathrm{HCHO}$ oxidation pathway in the cytosol. In this pathway, $\mathrm{HCHO}$ reacts non-enzymatically with a reduced form of GSH to generate $S$-hydroxymethyl glutathione ( $S$-HMG). Thereafter, $S$-HMG is used as a substrate for GSH-dependent formaldehyde dehydrogenase (FLD) to yield $S$-formylglutathione (S-FG). S-FG is then hydrolyzed to formate and GSH by $S$-formylglutathione hydrolase. Finally, formate is oxidized to $\mathrm{CO}_{2}$ by formate dehydrogenase. We have elucidated the physiological importance of the enzymes involved in the detoxification of $\mathrm{HCHO}$ and $\mathrm{H}_{2} \mathrm{O}_{2}$ by cloning and disrupting the corresponding genes in $C$. boidinii. ${ }^{9-14)}$ Furthermore, we found recently that the GSH redox system, including GSH biosynthesis and regeneration reactions, plays critical roles in yeast methanol metabolism, viz., detoxification and oxidation of $\mathrm{HCHO}$ and protection against reactive oxygen species (ROS) derived from $\mathrm{H}_{2} \mathrm{O}_{2}$. In addition, a transcription factor termed Yap1p plays an important role in the upregulation of GSH-related enzymes in P. pastoris. ${ }^{15,16)}$ In that the PpYap1p-depleted strain proved sensitive to $\mathrm{H}_{2} \mathrm{O}_{2}$ but not to $\mathrm{HCHO}$, methylotrophic yeasts must have an alternative transcriptional regulatory system to controll the expression of the genes for $\mathrm{HCHO}$ resistance.

To date, in the budding yeast Saccharomyces cerevisiae, stress-inducible transcription factors Msn2p/ Msn4p and heat shock protein Ssa4p, and their carrier Msn5p, have been reported to play important roles in protecting cells from stress-induced cell death. ${ }^{17-21)}$ Among these factors, we focused on Msn5p, which belongs to the importin- $\beta$ family and participates in the nuclear export of several proteins. Msn5p is not only

\footnotetext{
To whom correspondence should be addressed. Division of Applied Life Sciences, Graduate School of Agriculture, Kyoto University, Kitashirakawa-Oiwake, Sakyo-ku, Kyoto 606-8502, Japan; Fax: +81-75-753-6454; E-mail: ysakai@kais.kyoto-u.ac.jp

Abbreviations: AOD, alcohol oxidase; Msn5p-YFP, CbMsn5p tagged with YFP at the C-terminus; DAPI, 4',6'-diamidino-2-phenylindole; FLD, formaldehyde dehydrogenase; GSH, glutathione; qRT-PCR, quantitative real-time PCR; ROS, reactive oxygen species; RPA, replication protein A; $\mathrm{S}$-FG, $S$-formylglutathione; $S$-HMG, $S$-hydroxymethyl glutathione; Zeor ${ }^{\mathrm{r}}$, zeocin-resistant
} 
Table 1. Oligonucleotide Primers Used in This Study

\begin{tabular}{ll}
\hline \hline \multicolumn{1}{c}{ Primer name } & \multicolumn{1}{c}{ 5'-sequence-3' $^{\prime}$} \\
\hline PpMSN5-up-PstI & AACTGCAGATTTGTTGTAAGACATTTTG \\
PpMSN5-up-KpnI & GGGGTACCAGTGGATCCCAGATATTCTC \\
PpMSN5-down-SacII & TCCCCGCGGGCAGATAACGGATGGAATAT \\
PpMSN5-down-PstI & AACTGCAGGAAGAGGAACCTTCTAACAG \\
CbMSN5-up-EcoRI & CGGAATTCCATCACCTTCTCTCAAGCAATCAC \\
CbMSN5-up-XhoI & CCGCTCGAGGTAGCCAATTCGACAACCCATTGTCTAA \\
CbMSN5-down-SacII & TCCCCGCGGGTAGACCCTGAATTATCAGCTTGGTC \\
CbMSN5-down-EcoRI & CGGAATTCCTTCGAGAAGATGACGACGACTAATATAGC \\
SacI-CbMSN5-Fw & CCGAGCTCATAGCTGTCTGTGGCTCGAGGT \\
SbfI-CbMSN5-Rv & AAACGGCCTGCAGGTTTTTTAAAAGATGCAATGAATTGTAAAAATGCATTTCTTCTTTG \\
PstI-YFP-Fw & AACTGCAGGTTTCTAAAGGTGAAGAATTATTC \\
NheI-YFP-Rv & CTAGCTAGCTTATTTATATAATTCATCCATACC \\
PpACT1-RT-Fw & GCCGGTAGAGATTTGACCGACTACTTGATG \\
PpACT1-RT-Rv & GTAAGTGGTTTGGTCGATACCAGAAGCCTC \\
PpAOX1-RT-Fw & CCAAAGAAGCCAAGTCACAAGATCTACCGT \\
PpAOX1-RT-Rv & ACACCAGCTTCGATACCGTTAGTGGCAAGA \\
PpFLD1-RT-Fw & TGAGTGCAGAGAGTGCAAGTTCTGTCTGTC \\
PpFLD1-RT-Rv & CCAATACATCCAGCACCAAACACACCGATC \\
\hline
\end{tabular}

The underlined nucleotide sequences are additional restriction enzyme recognition sequences.

involved in nuclear export of the proteins that function in regulating carbohydrate metabolism, cell stress response, signal transduction control, cell cycle control, and so on, but also in the nuclear import of replication protein A (RPA), which is involved in DNA replication, DNA repair, and recombination. ${ }^{22)}$ Disruption of ScMSN5 caused lower survival rates than that of wildtype cells when cells were exposed to heat, starvation, ethanol, and oxidative stress conditions. ${ }^{21)}$ Thus Msn5p is required for responses to various kinds of stress.

Here we studied the physiological function of Msn5p in the methylotrophic yeast by gene disruption analysis and observation of the subcellular localization of Msn5p. Our results provide insight into the defense mechanism against $\mathrm{HCHO}$.

\section{Materials and Methods}

Strains, media, and cultivation conditions. Haploid strain C. boidinii S2 was used as the wild-type strain. ${ }^{23)} C$. boidinii strain TK62 (ura3) was used as host for transformation. ${ }^{24)}$ P. pastoris PPY12 (arg4 his4) was used as the wild-type strain. ${ }^{25)}$ Escherichia coli DH10B (Takara Bio, Kyoto, Japan) and SA116 ${ }^{24)}$ were used for plasmid propagation.

The yeast strains were grown on either YPD medium ( $2 \%$ glucose, $2 \%$ Bacto peptone, and 1\% Bacto yeast extract) or YNB medium (0.67\% Yeast Nitrogen Base without amino acids), supplemented, when required, with appropriate amino acids $(200 \mu \mathrm{g} / \mathrm{mL}$ for arginine and $200 \mu \mathrm{g} / \mathrm{mL}$ for histidine) or Zeocin (Invitrogen, Carlsbad, CA) $(50 \mu \mathrm{g} / \mathrm{mL}$ or $100 \mu \mathrm{g} / \mathrm{mL})$. One or more of the following were used as carbon sources in YNB medium: $2 \%(\mathrm{wt} / \mathrm{vol})$ glucose, $2 \%(\mathrm{vol} / \mathrm{vol})$ glycerol, $1 \%$ (vol/vol) methanol, $1 \%$ (vol/vol) ethanol, $0.5 \%$ (wt/vol) methylamine, and $0.5 \%$ (wt/vol) choline. In plate assays, the medium was supplemented with $1 \mathrm{~mm} \mathrm{H}_{2} \mathrm{O}_{2}, 2 \mathrm{~mm} \mathrm{HCHO}$, or $9 \mathrm{~mm} \mathrm{HCHO}$. The initial $\mathrm{pH}$ of the medium was adjusted to 6.0. Cultivation was performed at $28^{\circ} \mathrm{C}$ under aerobic conditions with reciprocal shaking, and the growth of the yeast was monitored by measuring the optical density at $610 \mathrm{~nm}$. HCHO was monitored by the method of Nash. ${ }^{26)}$

E. coli was grown at $37^{\circ} \mathrm{C}$ in $\mathrm{LB}$ medium ( $1 \%$ tryptone, $0.5 \%$ yeast extract, and $0.5 \% \mathrm{NaCl})$, supplemented, when required, with ampicillin $(50 \mu \mathrm{g} / \mathrm{mL})$ or zeocin $(50 \mu \mathrm{g} / \mathrm{mL})$.

Construction of CbMSN5 and PpMSN5 deletion strains. A deletion cassette for the CbMSN5 gene was constructed as follows: Primer pairs CbMSN5-up-EcoRI/CbMSN5-up-XhoI and CbMSN5-down-SacII/ CbMSN5-down-EcoRI (Table 1) were used to amplify $1.0-\mathrm{kb}$ and $1.1-\mathrm{kb}$ fragments using genomic DNA as template. Three fragments, the 1.0-kb EcoRI-XhoI fragment, the 1.1-kb SacII-EcoRI fragment, and the 4.1-kb XhoI-SacII fragment of plasmid $\mathrm{SK}^{+}-\mathrm{Zeo}^{r}$ (for C. boidinii ${ }^{27)}$ ) harboring $\mathrm{Zeo}^{r}$ gene, were ligated, yielding CbMSN5 disruption vector pDCbmsn5. pDCbmsn5 was digested with EcoRI, and then the 6.2-kb fragment was introduced into $C$. boidinii TK62 using a modified version of the lithium acetate method. ${ }^{24)}$ Zeocinresistant colonies were selected on YPD medium supplemented with zeocin. Disruption of the CbMSN5 gene was confirmed by Southern blot analysis using BglII digested genomic DNA from the transformants and a 1.0-kb EcoRI-XhoI PCR fragment from the coding region of the CbMSN5 gene as probe. The Cbmsn5 $\Delta$ strain was converted to uracil prototrophy by transforming it with plasmid pNOTeI, which contains the CbURA3 coding region. ${ }^{28)}$ The nucleotide sequence of CbMSN5 was deposited in DDBJ under accession no. AB669026.

In a similar way, a deletion cassette for the PpMSN5 gene (PAS_chr4_0654) was constructed as follows: Two fragments of the PpMSN5 gene $(0.98-\mathrm{kb}$ and $0.97-\mathrm{kb})$ were amplified by PCR with primer pairs PpMSN5-up-PstI/PpMSN5-up-KpnI and PpMSN5-downSacII/PpMSN5-down-PstI (Table 1) respectively with genomic DNA as template. The PCR products were recombined as PstI-KpnI and SacII-PstI fragments respectively and ligated with the 4.9-kb KpnISacII fragment of plasmid $\mathrm{SK}^{+}-Z e o^{r}$, which includes the zeocinresistant $\left(Z_{e o}{ }^{r}\right)$ gene, yielding PpMSN5 disruption cassette pDmsn5. After linearization with PstI, pDPpmsn5 was used to transform the wild-type strain by electroporation. Zeocin-resistant colonies were selected on YPD medium supplemented with zeocin. Disruption of the PpMSN5 gene was confirmed by Southern blot using ClaI-XbaIdigested genomic DNA from the transformants and a $0.98-\mathrm{kb}$ PstIKpnI PCR fragment from the coding region of the PpMSN5gene as probe.

Construction of strains expressing the ScPHO5 gene under the control of various promoters. Plasmids pAPU ${ }^{29)}$ and $\mathrm{pFLPU}$ (Yurimoto and Sakai, unpublished results), harboring the S. cerevisiae PHO5 gene, encoding acid phosphatase, under the control of the gene promoters of $C$. boidinii AOD1 and FLD1 respectively, were introduced into the Cbmsn5 $\Delta$ ura 3 strain. Integration of the plasmids into the genomes of the transformants was analyzed by Southern blotting of EcoRI-digested chromosomal DNA with the BamHI-PstI fragment of the URA3 gene as probe (data not shown). Transformants that showed a single integration event of pAPU or pFLPU at the ura3 locus in Cbmsn5 $\Delta$ were isolated, and were named APCbmsn5 $\Delta$ and FLPCbmsn $5 \Delta$ respectively. Acid phosphatase activity was measured as described previously. ${ }^{30)}$

Quantitative real-time PCR ( $q R T-P C R)$. Cells were grown aerobically to exponential phase in SM medium. Total RNAs was isolated from the cells with an RNeasy Mini Kit (Qiagen, Tokyo), followed by 
on-column DNase digestion. cDNAs were synthesized from $1 \mu \mathrm{g}$ of total RNA with Random Primers (Promega, Madison, WI) and ReverTra Ace (Toyobo, Osaka, Japan). After reverse transcription for $50 \mathrm{~min}$ at $42^{\circ} \mathrm{C}$, samples were heated for $5 \mathrm{~min}$ at $99^{\circ} \mathrm{C}$ to terminate the reaction, and $0.5 \mu \mathrm{L}$ of RNase $\mathrm{H}$ was added. qRT-PCR was performed in $20-\mu \mathrm{L}$ mixtures in glass capillary tubes in a LightCycler (Roche Diagnostics, Tokyo). The PCR cycling reaction was performed with $1 \times$ SYBR Premix Ex Taq (Takara Bio, Kyoto) according to the following parameters: first cycle, $10 \mathrm{~min}$ of denaturation at $95^{\circ} \mathrm{C}$; second cycle with 40 repetitions, $95^{\circ} \mathrm{C}$ for $20 \mathrm{~s}, 55^{\circ} \mathrm{C}$ for $20 \mathrm{~s}$, and $72^{\circ} \mathrm{C}$ for $20 \mathrm{~s}$ (all temperature transitions, $20^{\circ} \mathrm{C} \mathrm{s}^{-1}$ ). The primers used in the reactions are listed in Table 1. As negative control, PCR was done without ReverTra Ace. The relative abundance of mRNAs was standardized against the levels for PpACT1.

Construction of a strain expressing CbMsn5p and YFP-fused protein. To visualize the localization of CbMsn5p, a strain expressing the CbMsn5p-YFP fusion protein was constructed as follows: First, the CbMSN5 coding region and promoter region were amplified by PCR with primers SacI-MSN5-Fw and SbfI-MSN5-Rv, with genomic DNA as template. Then PCR was performed with primers PstI-YFP-Fw and NheI-YFP-Rv, with pTYT as the template. ${ }^{30)}$ The 5.4-kb SacI-SpeI fragment of pAPU, the 4.7-kb SacI-SbfI fragment of the promoter and coding regions of CbMSN5, and the 0.7-kb PstI-NheI fragment of the amplified YFP coding region were ligated to yield pCbMsn5-YFP. pCbMsn5-YFP was linearized with EcoRV and used to transform the Cbmsn5 $\Delta$ ura3 strain. The resulting strain was named CbMsn5p-YFP. Fluorescence microscopy and nuclear staining were performed as follows: CbMsn5p-YFP cells were harvested after exposure to stress conditions or non-stress conditions for $30 \mathrm{~min}$, washed once, and fixed with $1 \mathrm{~mL}$ of $70 \%$ ethanol for $30 \mathrm{~min}$ at room temperature. The fixed cells were washed twice, resuspended in $150 \mu \mathrm{L}$ of sterilized water, and stained with $150 \mu \mathrm{L}$ of $0.125 \mu \mathrm{g} / \mathrm{mL}$ DAPI (4',6'-diamidino-2-phenylindole) solution. After $10 \mathrm{~min}$ of incubation, fluorescence was observed under a fluorescence-inverted microscope (IX70; Olympus, Tokyo), as described previously. ${ }^{16)}$

\section{Results}

Cloning and characterization of the $\mathrm{C}$. boidinii and P. pastoris homologs of S. cerevisiae Msn5p

A search of the $C$. boidinii and $P$. pastoris genome databases revealed the presence of putative homologs of S. cerevisiae Msn5p, designated CbMsn5p and PpMsn5p respectively. CbMsn5p and PpMsn5p consisted of 1,233 and 1,239 amino acid residues respectively, and were $58.3 \%$ identical and $84.3 \%$ similar to each other. The deduced amino acid sequence of CbMsn5p was $41.0 \%$ identical and $74.0 \%$ similar to that of ScMsn5p, and the deduced amino acid sequence of PpMsn5p was $39.7 \%$ identical and $75.1 \%$ similar to that of ScMsn5p.

Physiological characterization of the C. boidinii msn5 $\Delta$ and $\mathrm{P}$. pastoris msn5 $\Delta$ strains

To analyze the physiological significance of CbMsn5p and PpMsn5p, we constructed MSN5 genedisrupted strains of $C$. boidinii and $P$. pastoris in which part of the ORF of the corresponding gene was replaced with the Zeor gene (Fig. 1; for details see "Materials and Methods"). We compared the growth of the wildtype and MSN5 gene-disrupted strains on various carbon and nitrogen sources. The growth of the C. boidinii wild-type and Cbmsn5 $\Delta$ strains on glucose or glycerol as sole carbon source was closely similar (data not shown). The Cbmsn5 strain exhibited retarded growth on methanol and on ethanol as sole carbon source as compared to the wild-type strain (Fig. 2A and B). In order to determine whether growth retardation was due to $\mathrm{HCHO}$ toxicity, the concentra-

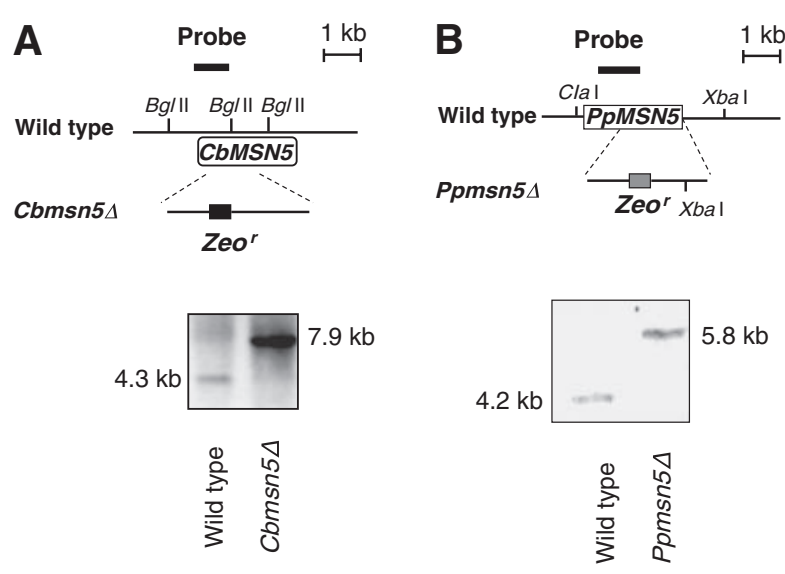

Fig. 1. One-Step Disruption of MSN5 Genes in C. boidinii and P. pastoris.

A, Physical map and gene disruption of CbMSN5. B, Physical map and gene disruption of PpMSN5. The probes used for Southern analysis are marked by bold lines.

tions of $\mathrm{HCHO}$ in the culture medium of the wild-type and Cbmsn5 cultures grown on methanol were determined (Fig. 2A). With the wild-type strain, $0.33 \mathrm{~mm} \mathrm{HCHO}$ accumulated after $26 \mathrm{~h}$, but thereafter rapidly disappeared. With the Cbmsn $5 \Delta$ strain, the $\mathrm{HCHO}$ level gradually increased until $30 \mathrm{~h}$ and then gradually decreased. These results suggest that CbMsn5p is involved in $\mathrm{HCHO}$ consumption during growth on methanol. When methylamine or choline, which are metabolized with formation of $\mathrm{HCHO}$, was used as sole nitrogen source together with glucose as a carbon source, the Cbmsn5 $\Delta$ strain also exhibited retarded growth as compared to the wild-type strain (Fig. 2C and D). In the case of P. pastoris, the Ppmsn5 $\triangle$ strain grew as well on $1 \%$ methanol as the wild-type strain (Fig. 3A), but the Ppmsn5 $\Delta$ strain showed retarded growth as compared to the wild-type strain on 3\% methanol (Fig. 3B). This suggests that Msn5p plays an important role in methylotrophic yeasts when the cells are grown on substrates that result in the formation of $\mathrm{HCHO}$, and that Msn5p is more critical for growth on methanol in $C$. boidinii than P. pastoris.

Msn5p was not involved in the transcriptional activation of methanol-inducible genes

To test whether disruption of MSN5 affects the transcriptional activation of methanol-inducible genes, the expression levels of methanol-inducible genes were analyzed using a promoter-reporter assay for $C$. boidinii $A O D 1$ and FLDI and qRT-PCR analysis of P. pastoris $A O X 1$ and FLDI.

We constructed strains expressing the ScPHO5 gene under the promoters for $C b A O D 1$ and $C b F L D 1$. Both genes are induced not only by methanol but also by HCHO. ${ }^{12)}$ In glucose medium, expression of both genes was completely repressed, and both promoters exhibited basal levels of transcriptional activities in the wild-type and Cbmsn5 $\Delta$ strains (Table 2). Promoter activities in the Cbmsn $5 \Delta$ cells induced by both $0.7 \%$ methanol and $3 \mathrm{~mm} \mathrm{HCHO}$ were higher than those of the wild-type strain (Table 2). However, when the cells were induced by $9 \mathrm{~mm}$ HCHO (the wild-type strain grew in a medium containing up to $9 \mathrm{~mm} \mathrm{HCHO}$ ), no reporter activity in 

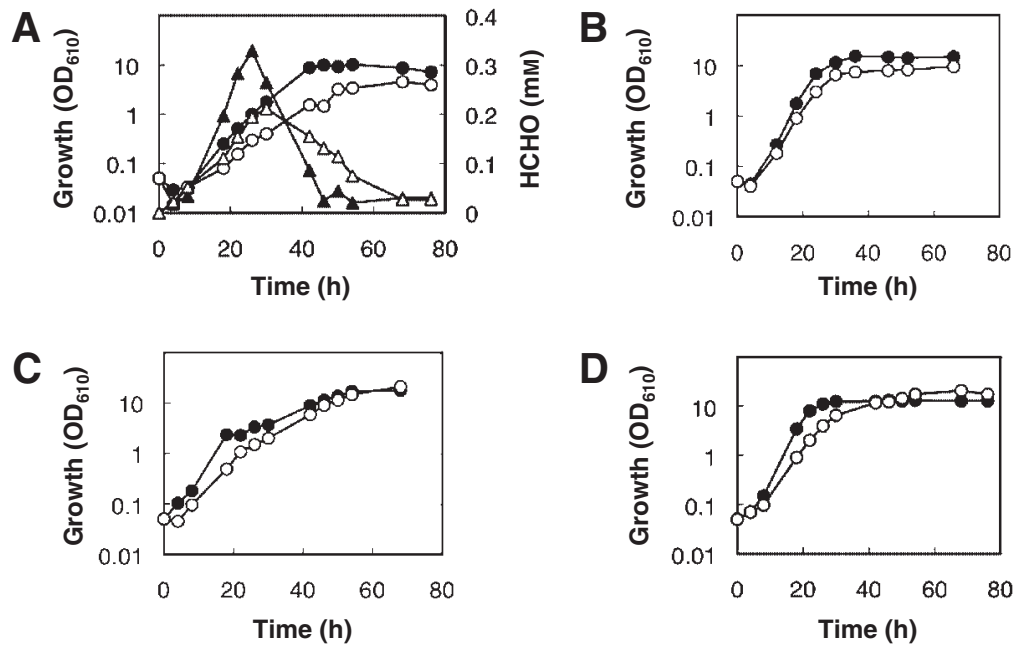

Fig. 2. Growth of the Wild-Type and Cbmsn5 $\Delta$ Strains on Various Carbon and Nitrogen Sources, and the HCHO Concentration in the Medium. Cells were grown in YNB medium with the carbon and nitrogen sources indicated: A, $1 \%$ methanol; B, $1 \%$ ethanol; C, $2 \%$ glucose and $0.5 \%$ choline; and D, $2 \%$ glucose and $0.5 \%$ methylamine. Solid and hollow circles represent the growth of the wild-type and Cbmsn5 $\Delta$ strains respectively; solid and hollow triangles represent the $\mathrm{HCHO}$ concentration in the medium with the wild-type and $\mathrm{Cbmsn5} \Delta$ strains respectively.
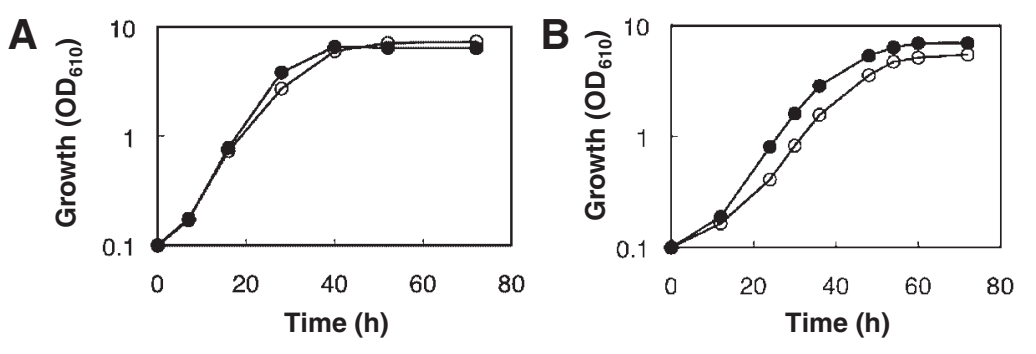

Fig. 3. Growth of the Wild-Type and Ppmsn5 $\Delta$ Strains in YNB Medium with 1\% Methanol (A) and 3\% Methanol (B). Solid and hollow circles represent the wild-type and Ppmsn5 $\Delta$ strains respectively.

Table 2. Levels of Acid Phosphatase Activity under the AOD1 and FLD1 Promoters in the Cbmsn5 $\triangle$ Strain

\begin{tabular}{|c|c|c|c|}
\hline \multirow{2}{*}{ Carbon source } & \multirow{2}{*}{ Strain } & \multicolumn{2}{|c|}{ Reporter activity $^{\mathrm{a}}$} \\
\hline & & $P_{A O D 1}$ & $P_{F L D I}$ \\
\hline \multirow[t]{2}{*}{ Glucose } & Wild type & $0.79 \pm 0.05$ & $1.68 \pm 0.09$ \\
\hline & Cbmsn5 $\Delta$ & $1.09 \pm 0.09$ & $2.28 \pm 0.04$ \\
\hline \multirow[t]{2}{*}{ Methanol } & Wild type & $24.5 \pm 0.23$ & $27.8 \pm 1.32$ \\
\hline & Cbmsn5 $\Delta$ & $66.0 \pm 5.97$ & $195 \pm 11.0$ \\
\hline \multirow[t]{2}{*}{$\mathrm{HCHO}(3 \mathrm{~mm})$} & Wild type & $57.9 \pm 1.33$ & $70.9 \pm 2.81$ \\
\hline & Cbmsn5 $\Delta$ & $117 \pm 18.2$ & $176 \pm 2.81$ \\
\hline \multirow[t]{2}{*}{ HCHO (9 mм) } & Wild type & $1.74 \pm 0.10$ & $0.60 \pm 0.25$ \\
\hline & Cbmsn5 & N.D. & N.D. \\
\hline
\end{tabular}

a The level of acid phosphatase activity is expressed as $\mathrm{U}^{\mathrm{a}} \mathrm{OD}_{660}$ on the respective carbon source. The means of three independent experiments are shown. The wild-type and Cbmsn5 $\Delta$ strains were incubated in the presence of $2 \%$ glucose, $0.7 \%$ methanol, $3 \mathrm{~mm} \mathrm{HCHO}$, or $9 \mathrm{~mm} \mathrm{HCHO}$ for 8 h. N.D., Activity could not be detected.

the Cbmsn5 $\Delta$ strain was detected, because the cells were dead at $9 \mathrm{~mm}$ HCHO. Similar results were observed for the expression levels of the PpAOXI and PpFLDI genes in the cells induced by methanol, as determined by qRTPCR analysis (Table 3). This indicates that Msn5p is not involved in the transcriptional activation of methanol/ $\mathrm{HCHO}$-inducible genes, and participates in a defense mechanism against HCHO. In the Msn5p-depleted strains, the intracellular level of HCHO might be higher than in the wild-type strain, and in that case, the accumulated $\mathrm{HCHO}$ more strongly induces the expression of HCHO-inducible genes.
Table 3. Transcriptional Levels of $P p A O X 1$ and $P p F L D 1$ in the Ppmsn5 Strain

\begin{tabular}{cccc}
\hline \hline \multirow{2}{*}{$\begin{array}{c}\text { Carbon } \\
\text { source }\end{array}$} & Strain & \multicolumn{2}{c}{ Relative transcriptional level } \\
\cline { 3 - 4 } & & PpAOX1 & PpFLD1 \\
\hline \multirow{2}{*}{ Methanol } & Wild type & $100 \%$ & $100 \%$ \\
& Ppmsn5 & $143 \%$ & $201 \%$ \\
\hline
\end{tabular}

The $P$. pastoris wild-type and Ppmsn5 $\Delta$ strains were incubated in the presence of $0.7 \%$ methanol for $3 \mathrm{~h}$. The relative abundance of mRNAs was standardized against the level of PpACT1.

Msn5p was involved in the defense mechanism against $\mathrm{HCHO}$, but not against $\mathrm{H}_{2} \mathrm{O}_{2}$

To elucidate the functional consequences of depletion of Msn5p on the $\mathrm{HCHO}$ or $\mathrm{H}_{2} \mathrm{O}_{2}$ stress response system, the Cbmsn5 $\Delta$ and Ppmsn5 $\Delta$ strains were plated on YNB-glucose agar plates containing an appropriate concentration of $\mathrm{HCHO}$ or $\mathrm{H}_{2} \mathrm{O}_{2}$. The Cbmsn $5 \Delta$ strain exhibited higher sensitivity to $\mathrm{HCHO}$ than the wild-type strain (Fig. 4A). On the other hand, the Cbmsn5 $\Delta$ strain was observed to become hyper-resistant to $1 \mathrm{~mm} \mathrm{H}_{2} \mathrm{O}_{2}$. Similarly, the Ppmsn5 $\Delta$ strain was more sensitive to $\mathrm{HCHO}$, but less sensitive to $\mathrm{H}_{2} \mathrm{O}_{2}$ (Fig. 4B) as compared with the wild-type strain. This suggests that Msn5p is involved in the defense mechanism against $\mathrm{HCHO}$, but not against $\mathrm{H}_{2} \mathrm{O}_{2}$.

Subcellular localization of CbMsn5p-YFP

In $S$. cerevisiae, $\mathrm{ScMsn} 5 \mathrm{p}$ is concentrated in the nuclei under non-stress conditions, but diffuses into the 
A

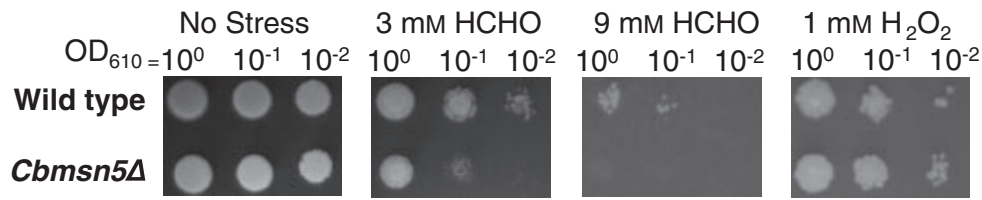

B

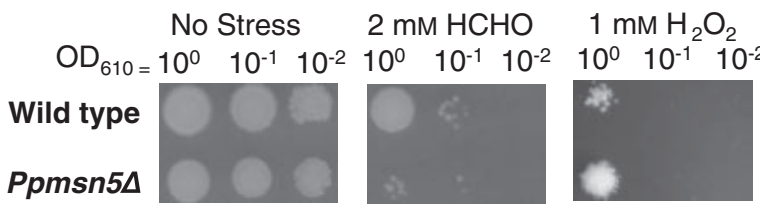

Fig. 4. Cell Viability in Medium Containing $\mathrm{HCHO}$ or $\mathrm{H}_{2} \mathrm{O}_{2}$.

A, The $C$. boidinii wild-type and Cbmsn5 $\Delta$ strains were incubated in YNB-glucose medium containing $3 \mathrm{~mm} \mathrm{HCHO,} 9 \mathrm{~mm} \mathrm{HCHO}$, or $1 \mathrm{mM}$ $\mathrm{H}_{2} \mathrm{O}_{2}$, and then 10-fold serial dilutions were spotted on YNB-glucose agar plates, which were incubated for $2 \mathrm{~d}$ at $28^{\circ} \mathrm{C}$. B, The $P$. pastoris wild-

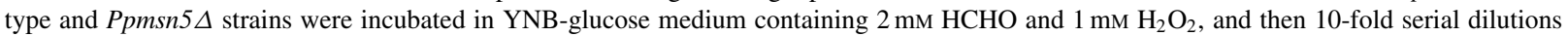
were spotted on YNB-glucose agar plates, which were incubated for $2 \mathrm{~d}$ at $28^{\circ} \mathrm{C}$.

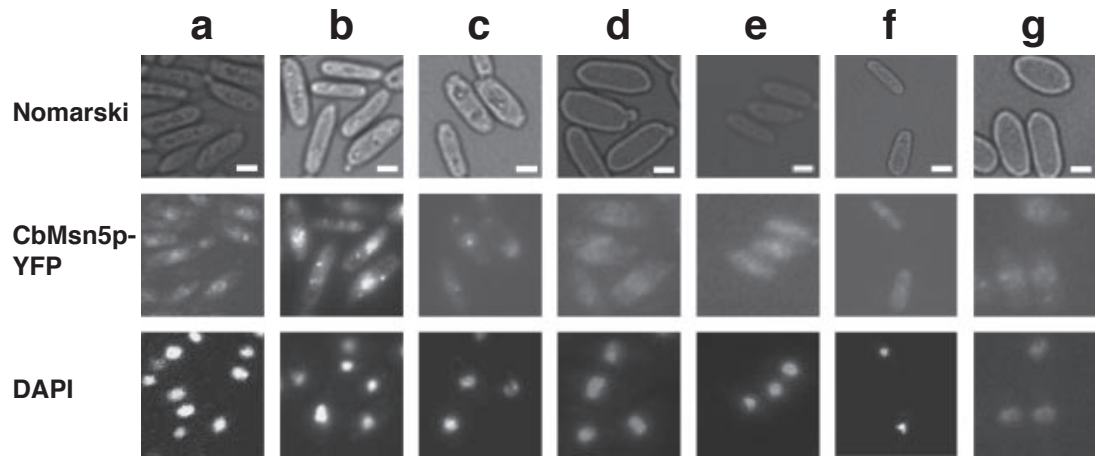

Fig. 5. Subcellular Localization of CbMsn5p-YFP.

CbMsn5p-YFP was expressed under the control of the native promoter in the Cbmsn5 $\Delta$ strain. CbMsn5p-YFP expressing cells were incubated on YNB medium containing various carbon sources, and YFP fluorescence and DAPI-stained nuclei were observed using fluorescence microscopy. a, $2 \%$ glucose (at mid-log phase); b, $1 \%$ methanol (mid-log phase); c, $1 \%$ methanol ( 30 min incubation); d, $2 \%$ glucose with 3 mM $\mathrm{HCHO}$ (30 min incubation); e, $2 \%$ glucose with $9 \mathrm{~mm} \mathrm{HCHO} \mathrm{(30} \mathrm{min} \mathrm{incubation);} \mathrm{f,} 10 \%$ ethanol (30 min incubation); g, $2 \%$ glucose with $1 \mathrm{~mm}$ $\mathrm{H}_{2} \mathrm{O}_{2}$ (30 min incubation). Bar, $1 \mu \mathrm{m}$.

cytosol upon exposure to various stresses, e.g., ethanol, heat, starvation, and severe oxidative stress. ${ }^{21)}$ To investigate the subcellular localization of CbMsn5p, we expressed a recombinant CbMsn5p tagged with YFP at the C-terminus (Msn5p-YFP) under the control of the native promoter in the Cbmsn5 $\Delta$ strain.

The growth rate of the Cbmsn5 $\Delta$ strain expressing Msn5p-YFP on glucose with $3 \mathrm{~mm}$ HCHO was similar to that of the wild-type strain (data not shown), indicating that fusion of the YFP protein to Msn5p did not interfere with its native function. When cells expressing CbMsn5p-YFP were grown on glucose or methanol to mid-log phase, or when the cells were exposed to $1 \%$ methanol for $30 \mathrm{~min}$, the fluorescence of CbMsn5p-YFP was partially concentrated in the nucleus, as shown by staining with DAPI (Fig. 5a, b, and c). When the cells were exposed to $3 \mathrm{~mm} \mathrm{HCHO}$, CbMsn5p-YFP accumulated in the nucleus (Fig. 5d). However, when the cells were exposed to 6-9 mM HCHO, CbMsn5p-YFP diffused into the cytosol (Fig. 5e). The distribution of ScMsn5p has been reported to be correlated with the concentration of ethanol. ${ }^{21)}$ CbMsn5p-YFP diffused into the cytosol when the cells were exposed to $10 \%$ ethanol (Fig. 5f), but CbMsn5p-YFP accumulated in the nucleus when the cells were exposed to $1 \mathrm{mM} \mathrm{H}_{2} \mathrm{O}_{2}$ (Fig. $5 \mathrm{~g}$ ). When the cells were exposed to $5 \mathrm{mM} \mathrm{H}_{2} \mathrm{O}_{2}$, CbMsn5pYFP still accumulated in the nucleus (data not shown). This suggests that CbMsn5p responds to HCHO stress and ethanol stress but not to $\mathrm{H}_{2} \mathrm{O}_{2}$ stress.

\section{Discussion}

In yeast methanol metabolism, two toxic compounds, $\mathrm{HCHO}$ and $\mathrm{H}_{2} \mathrm{O}_{2}$, are generated by alcohol oxidase. In previous studies, we determined the physiological functions of each of the enzymes involved in the detoxification of these compounds and the importance of the GSH redox system in methylotrophic yeasts. ${ }^{3)}$ The GSH redox system is regulated by oxidative stress regulator Yap1p, and a Ppyap1 $\Delta$ strain showed a prolonged lag phase during growth on methanol, but this strain did not show sensitivity to HCHO. ${ }^{15,16)}$ Thus it was clear that the cellular response to ROS derived from $\mathrm{H}_{2} \mathrm{O}_{2}$ is regulated by transcription factor Yap1p, but the molecular mechanism of the cellular response to $\mathrm{HCHO}$ remained unknown.

In this study, we focused on Msn5p, a nuclear exporter of the importin- $\beta$ family, in methylotrophic yeasts. MSN5 was originally isolated as a multicopy suppressor in $S$. cerevisiae mutants defective in Snflp, which is a protein kinase and a key regulator of cell growth and a metabolic sensor. ${ }^{31,32)}$ Although multiple functions for Msn5p have been elucidated, there has been no report as to whether Msn5p participates in the $\mathrm{HCHO}$ resistance mechanism. In the present study, we found that Msn5p in the methylotrophic yeasts was involved in the cellular defense mechanism against $\mathrm{HCHO}$ by gene disruption analysis and observation of the subcellular localization of Msn5p. 
In $S$. cerevisiae, the subcellular localization of Msn5p is controlled by stress and carbon source in a Snf1pdependent manner. ${ }^{21,33)} \mathrm{ScMsn} 5 \mathrm{p}$ is concentrated in the nuclei under non-stress conditions, but appears in the cytoplasm under stress conditions, such as exposure to ethanol, heat, starvation, or oxidative stress. ${ }^{21)}$ YFPtagged CbMsn5p was diffuse in the cytoplasm when the cells were treated with high concentrations of $\mathrm{HCHO}$ (6-9 mM) and ethanol (10\%), but fluorescence was concentrated in the nuclei following treatment with $\mathrm{H}_{2} \mathrm{O}_{2}$ (1-5 mM). Taken together with the results as to the sensitivity of the msn5 $\Delta$ strains to $\mathrm{HCHO}$ and $\mathrm{H}_{2} \mathrm{O}_{2}$, it is clear that Msn5p participates in the response to $\mathrm{HCHO}$ stress but not directly in $\mathrm{H}_{2} \mathrm{O}_{2}$ stress in the methylotrophic yeasts. Interestingly, the behavior of CbMsn5pYFP was similar when the cells were exposed to $\mathrm{HCHO}$ and ethanol, indicating that the cellular responses to $\mathrm{HCHO}$ and ethanol stress might be regulated in part by the same pathway. In $S$. cerevisiae, molecular responses to ethanol and acetaldehyde stress have been studied, ${ }^{34,35)}$ but no effect of acetaldehyde or $\mathrm{HCHO}$ on ScMsn5p has been reported.

Msn5p mediates transcriptional regulation by exporting transcription factors, such as Miglp and Pho4p, in S. cerevisiae. ${ }^{36,37)}$ Hence it is possible that Msn5p in the methylotrophic yeasts functions in the transcriptional regulation of genes encoding methanol metabolism. However, our results indicate that Msn5p is not involved in the transcriptional activation of AODI and FLDI in methylotrophic yeasts (Tables 2 and 3). The transcription levels of these genes in the msn5 $\Delta$ strains were significantly higher than those in the wild-type strain. Their promoters might be more strongly activated by higher levels of $\mathrm{HCHO}$ by an unknown mechanism in the $m s n 5 \Delta$ strain.

In addition to nuclear export, ScMsn5p mediates the nuclear import of replication protein A (RPA), which is required for multiple aspects of DNA metabolism, including DNA replication, DNA repair, and recombination. $22,38-40)$ It is well known that $\mathrm{HCHO}$ is nonspecifically reactive with nucleic acids, and that exposure to $\mathrm{HCHO}$ results in damaged DNA or inhibition of DNA repair. Hence we speculate that $\mathrm{HCHO}$ temporarily accumulates in cells, causing toxic effects on the DNA and/or DNA repair, and that RPA is required to protect cells from $\mathrm{HCHO}$ toxicity. Disruption of MSN5 can result in a reduced rate of localization of RPA in the nucleus and increased cell sensitivity to HCHO. To substantiate this hypothesis, further experiments are needed.

Our findings perhaps shed light on the details of the defense mechanism against $\mathrm{HCHO}$, and might help to employ the methylothophic yeast efficiently to produce foreign protein at high $\mathrm{HCHO}$ concentrations. To our knowledge, this is the first report of this new role for Msn5p, which is indirectly or directly involved in the response to $\mathrm{HCHO}$ stress in methylothophic yeasts.

\section{Acknowledgments}

This research was supported in part by Grant-in-Aid for Scientific Research (B) (22380052 to Y.S. and 22310046 to H.Y.) from the Japan Society for the Promotion of Science. It was also supported by CREST, JST.

\section{References}

1) Yurimoto H, Sakai Y, and Kato N, "Hansenula polymorphaBiology and Applications_," ed. Gellissen G, Wiley-VCH, Weinheim, pp. 61-75 (2002).

2) Yurimoto H, Kato N, and Sakai Y, Chem. Rec., 5, 367-375 (2005).

3) Yurimoto H, Oku M, and Sakai Y, Int. J. Microbiol., 2011, 101298 (2011).

4) Cregg JM, Cereghino JL, Shi J, and Higgins DR, Mol. Biotechnol., 16, 23-52 (2000).

5) Gellissen G, Appl. Environ. Microbiol., 54, 741-750 (2000).

6) Sakai Y, Tani Y, and Kato N, J. Mol. Cat. B: Enzymatic, 6, 161173 (1999).

7) Yurimoto H, Biosci. Biotechnol. Biochem., 73, 793-800 (2009).

8) Yurimoto H and Sakai Y, Biotechnol. Appl. Biochem., 53, 8592 (2009).

9) Horiguchi $\mathrm{H}$, Yurimoto $\mathrm{H}$, Goh $\mathrm{T}$, Nakagawa $\mathrm{T}$, Kato $\mathrm{N}$, and Sakai Y, J. Bacteriol., 183, 6372-6383 (2001).

10) Sakai Y, Murdanoto AP, Konishi T, Iwamatsu A, and Kato N, J. Bacteriol., 179, 4480-4485 (1997).

11) Horiguchi H, Yurimoto H, Kato N, and Sakai Y, J. Biol. Chem., 276, 14279-14288 (2001).

12) Lee B, Yurimoto H, Sakai Y, and Kato N, Microbiology, 148, 2697-2704 (2002).

13) Yurimoto H, Lee B, Yano T, Sakai Y, and Kato N, Microbiology, 149, 1971-1979 (2003).

14) Yurimoto H, Lee B, Yasuda F, Sakai Y, and Kato N, Yeast, 21, 341-350 (2004).

15) Yano T, Takigami E, Yurimoto H, and Sakai Y, Eukaryot. Cell, 8, 540-549 (2009).

16) Yano $\mathrm{T}$, Yurimoto $\mathrm{H}$, and Sakai $\mathrm{Y}$, Biosci. Biotechnol. Biochem., 73, 1404-1411 (2009).

17) Estruch F, FEMS Microbiol. Rev., 24, 469-486 (2000).

18) Gasch AP, Spellman PT, Kao CM, Carmel-Harel O, Eisen MB, Storz G, Botstein D, and Brown PO, Mol. Biol. Cell, 11, 42414257 (2000).

19) Schuller C, Mamnun YM, Mollapour M, Krapf G, Schuster M, Bauer BE, Piper PW, and Kuchler K, Mol. Biol. Cell, 15, 706720 (2004).

20) Durchschlag E, Reiter W, Ammerer G, and Schuller C, J. Biol. Chem., 279, 55425-55432 (2004).

21) Quan X, Tsoulos P, Kuritzky A, Zhang R, and Stochaj U, Mol. Microbiol., 62, 592-609 (2006).

22) Yoshida K and Blobel G, J. Cell Biol., 152, 729-740 (2001).

23) Tani Y, Sakai Y, and Yamada H, Agric. Biol. Chem., 49, 26992706 (1985).

24) Sakai Y, Kazarimoto T, and Tani Y, J. Bacteriol., 173, 74587463 (1991).

25) Sakai Y, Koller A, Rangell LK, Keller GA, and Subramani S, J. Cell Biol., 141, 625-636 (1998).

26) Nash T, Biochem. J., 55, 416-421 (1953).

27) Sasano Y, Yurimoto H, and Sakai Y, J. Biosci. Bioeng., 104, 86-89 (2007).

28) Sakai Y, Akiyama M, Kondoh H, Shibano Y, and Kato N, Biochim. Biophys. Acta, 1308, 81-87 (1996).

29) Yurimoto H, Komeda T, Lim C, Nakagawa T, Kondo K, Kato N, and Sakai Y, Biochim. Biophys. Acta, 1493, 56-63 (2000).

30) Sasano Y, Yurimoto H, Yanaka M, and Sakai Y, Eukaryot. Cell, 7, 527-536 (2008).

31) Estruch F and Carlson M, Nucleic Acids Res., 18, 6959-6964 (1990).

32) Estruch F and Carlson M, Mol. Cell. Biol., 13, 3872-3881 (1993).

33) Quan X, Yu J, Bussey H, and Stochaj U, Biochim. Biophys. Acta, 1773, 1052-1061 (2007).

34) Aranda A, Querol A, and del Olmo M, Arch. Microbiol., 177, 304-312 (2002).

35) Aranda A and del Olmo M, Yeast, 20, 747-759 (2003).

36) Devit MJ and Johnston M, Curr. Biol., 9, 1231-1241 (1999).

37) Kaffmani A, Rankl NM, O'neilli EM, Huang LS, and O'shea EK, Nature, 396, 482-486 (1998).

38) Wold MS, Annu. Rev. Biochem., 66, 61-92 (1997).

39) Waga S and Stillman B, Annu. Rev. Biochem., 67, 721-751 (1998).

40) Iftode C, Daniely Y, and Borowiec JA, Crit. Rev. Biochem. Mol. Biol., 34, 141-180 (1999). 